\title{
Data Literacy Education für Studierende aller Fächer. Kompetenzziele, curriculare Integration und didaktische Ausgestaltung interdisziplinärer Lehr-Lern-Angebote
}

\section{Matthias Bandtel, Leonie Kauz und Natalia Weißker}

\section{Zusammenfassung}

Data Literacy ist eine zentrale Voraussetzung für die aktive Teilhabe an der digitalen Gesellschaft. Allerdings gibt es noch nicht für Studierende aller Fächer Angebote, die ihnen die Entwicklung dieser Schlüsselkompetenz ermöglichen. Der Beitrag diskutiert Herangehensweisen an die Definition von Lernzielen, Möglichkeiten der curricularen Integration und geeignete didaktische Ausgestaltungen von Lehr-Lernsettings. Anhand einer Fallstudie zum Mannheimer Modell Data Literacy Education (modal) und empirischen Befunden aus der Begleitforschung werden potenzielle Umsetzungsstrategien diskutiert. Ziel des Beitrags ist, Impulse und Handlungsempfehlungen für die Integration von Data Literacy Education in Lehre und Lernen für Studierende aller Fächer zu geben.

M. Bandtel ( $\square)$

Hochschulnetzwerk Digitalisierung der Lehre Baden-Württemberg (HND-BW), Karlsruhe, Deutschland

E-Mail: matthias.bandtel@kit.edu

L. Kauz · N. Weißker

Hochschule Mannheim, Mannheim, Deutschland

E-Mail:1.kauz@hs-mannheim.de

N. Weißker

E-Mail: n.weissker@hs-mannheim.de 


\section{Schlüsselwörter}

Data Literacy Education • Datenkompetenzen • Lehre • Lernen • Interdisziplinarität • Curriculare Integration • Didaktik • Evaluation • Kompetenzmessung • Future Skills

\section{Problemstellung:Teilhabe an der digitalen Gesellschaft ermöglichen}

Data Literacy gilt als zentrale Voraussetzung für die aktive Teilhabe und verantwortliche Mitgestaltung der digitalen Transformation (Schüller et al. 2019, S. 10). Dementsprechend wird der Bedarf einer verstärkten Förderung von Datenkompetenzen für Studierende aller Fächer aus unterschiedlichen Perspektiven artikuliert: Erstens melden Unternehmen aller Branchen eine steigende Nachfrage nach Arbeitnehmer*innen mit grundlegenden Datenkompetenzen an (Kirchherr et al. 2018). Zweitens erkennen mehr und mehr Hochschulen die Notwendigkeit, Studierenden die Entwicklung digitaler Schlüsselkompetenzen zu ermöglichen (Heidrich et al. 2018). Drittens fordern auch Studierende mit Nachdruck, Digital Literacy in die Curricula aller Fächer zu integrieren, um als mündige Bürger*innen in der digitalen Gesellschaft verantwortlich handeln zu können (Baumann et al. 2019, S. 2). Und viertens hat die Politik inzwischen den Ball aufgenommen. In der Digitalstrategie der Bundesregierung (2019) kommt dem Handlungsfeld digitale Kompetenz ein zentraler Stellenwert zu. Im gesamten Bildungssystem werden Maßnahmen angedacht, Fähigkeiten zu stärken, den digitalen Wandel zu gestalten (Bundesregierung 2019).

Auf den ersten Blick besteht also große Einigkeit hinsichtlich der Ziele. Doch beim genaueren Hinsehen stehen die Akteur*innen in Wirtschaft, Wissenschaft und Politik bei der Umsetzung noch am Anfang. Dieser Beitrag wirft einige Schlaglichter auf den aktuellen Stand des Diskurses über Data Literacy Education in der Hochschulbildung und zeigt mögliche Umsetzungsbeispiele auf. Zunächst wird der Frage nachgegangen, welche Kompetenzen Studierende aller Fächer entwickeln müssen, um an der digitalen Gesellschaft aktiv teilhaben und Veränderungen in Beruf und Gesellschaft verantwortlich mitgestalten zu können (Abschn. 2). Darauf aufbauend wird diskutiert, wie diese Lernziele in die Curricula möglichst aller Fächer integriert werden können respektive welche LehrLern-Settings und didaktischen Methoden die Entwicklung dieser Kompetenzen nachhaltig ermöglichen (Abschn. 3). Dabei werden insbesondere die Chancen 
und Herausforderungen interdisziplinärer Lehr-Lern-Formate ausgelotet (Abschn. 4). Eine Fallstudie zum Mannheimer Modell Data Literacy Education (,modal“) illustriert Möglichkeiten der operativen Umsetzung von Lernzielen, der curricularen Integration und didaktischen Feinjustierung im Hochschulkontext (Abschn. 5). Auf der Basis von Daten aus der Evaluation und Begleitforschung zu ,modal“ werden Wirksamkeit der Maßnahmen und Weiterentwicklungspotenziale diskutiert (Abschn. 6). Die wichtigsten Erkenntnisse werden abschließend noch einmal zusammengefasst (Abschn. 7).

\section{Kompetenzen und Lernziele}

In ihrer inzwischen zum Standardwerk avancierten Metastudie definiert die kanadische Forscher*innengruppe um Chantel Ridsdale Data Literacy als ,the ability to collect, manage, evaluate, and apply data, in a critical manner" (Ridsdale et al. 2015, S. 2). Um das Grundkonzept von Daten und die vier definitorischen Kernaufgaben herum entwickelt sie ein Kompetenzraster, das auf oberster Ebene die Felder Conceptual Framework, Data Collection, Data Management, Data Evaluation und Data Application umfasst (Ridsdale et al. 2015, S. 3). Diesen werden insgesamt 64 Fähigkeiten, Fertigkeiten und Kenntnisse zugeordnet (Ridsdale et al. 2015, S. 39 ff.), die wiederum auf drei unterschiedlichen Niveaustufen (Conceptual Competencies, Core Competencies und Advanced Competencies) verortet sind.

Die Systematik von Ridsdale et al. bildete den Ausgangspunkt für zahlreiche Folgestudien. Beispielsweise wurde sie aus zivilgesellschaftlicher Perspektive von Engel et al. (2019) aufgegriffen. In den Bibliothekswissenschaften nehmen unter anderem Pothier und Condon (2019) Bezug auf das Standardwerk. Operationalisierungen im Hochschulkontext haben zum Beispiel Lübcke und Wannemacher (2018) untersucht. Aufbauend auf den umfassenden Vorarbeiten legen Katharina Schüller und Kolleginnen (2019) ihrem Kompetenzraster ein Verständnis von Data Literacy als ,Cluster aller effizienten Verhaltensweisen und Einstellungen für die effektive Erfüllung sämtlicher Prozessschritte zur Wertschöpfung beziehungsweise Entscheidungsfindung aus Daten“ (Schüller et al. 2019, S. 23) zugrunde. Auf Basis einer Rekonstruktion der historischen Genese des Data-LiteracyBegriffs wird ein Kompetenzrahmen hergeleitet, der insgesamt 18 Kompetenzen einbezieht. Diese werden entlang eines zyklischen Prozessmodells der Datenwertschöpfung sechs Kompetenzfeldern zugeordnet: (A) Datenkultur etablieren, (B) Daten bereitstellen, (C) Daten auswerten, (D) Datenprodukte interpretieren, (E) Daten interpretieren und (F) Handeln ableiten. Dabei unterscheiden Schüller 
et al. (2019) produktive Prozessschritte (A bis C), die bei der Überführung von Daten zu Datenprodukten zum Einsatz kommen, von rezeptiven Prozessschritten (D bis F), die zur Dekodierung von Datenprodukten und der Rekonstruktion zugrunde liegender Daten erforderlich sind. In jedem Kompetenzfeld werden jeweils benötigtes Fachwissen, Fähigkeiten und Haltungen generisch beschrieben sowie orientiert am Europäischen Qualifikationsrahmen für lebenslanges Lernen aufsteigenden Komplexitätsniveaus zugerechnet (Schüller et al. 2019, S. 33 f.).

Diese Ansätze haben sich um die Systematisierung des Diskurses über Data Literacy in besonderer Weise verdient gemacht. Sie haben wesentliche Grundlagen für die curriculare und didaktische Umsetzung von Lehr-Lern-Angeboten zur Entwicklung von Datenkompetenzen geschaffen. Die Ableitung konkreter Lernziele sowie die Ausgestaltung spezifischer Lehr-Lern-Settings kann sich an diesen wichtigen Vorarbeiten orientieren (für ein Praxisbeispiel vgl. Abschn. 5).

\section{Curriculare Integration und didaktische Ausgestaltung}

Auf Grundlage ihrer Analyse bestehender Best Practices identifizieren Ridsdale et al. (2015) Strategien der curricularen Integration sowie idealtypische hochschuldidaktische Ansätze zur Förderung von Datenkompetenzen (Ridsdale et al. 2015, S. 19 ff.). Zunächst einmal werden die bereits vollen Curricula als größte Hürde für die flächendeckende Förderung von Datenkompetenzen für Studierende aller Fächer bestimmt. Einen möglichen Lösungsweg stellt die Integration von Datenkompetenzen in bestehende über- und außerfachliche Lehr-Lern-Angebote dar. Als Beispiele werden Methoden- oder Statistikkurse sowie Veranstaltungen zu Information respektive Visual Literacy genannt, weil hier Schnittmengen mit Data-Literacy-Kompetenzen gegeben sind (Ridsdale et al. 2015, S. 21). Mit Blick auf die didaktische Ausgestaltung fassen Ridsdale et al. (2015) folgende Gelingensbedingungen für nachhaltige Lehr-Lern-Modelle zusammen:

- Transparenz der Lernziele: Mehrwerte von Daten und Datenkompetenzen müssen von Anfang an erkennbar sein.

- Anwendungsorientiertes Lernen in Workshops und Laboren: Die praktische Erfahrung ermöglicht Studierenden, Fähigkeiten vollumfänglich zu entwickeln. Wichtige Gelingensbedingung ist die Freiheit, Prozesse und Methoden selbstorganisiert zu erkunden und zu erproben, dabei Fehler machen zu dürfen und aus den Erfahrungen zu lernen.

- Modularisiertes Lernen: Eine systematische sukzessive Staffelung oder iterative Verbindung der Lernziele ermöglicht Studierenden, neues Wissen und 
neue Fähigkeiten an zuvor entwickelte Kompetenzen anzuschließen. Auf diese Weise wird ein vertieftes Verständnis von ganzheitlichen Prozessen entwickelt, statt Lerninhalte lediglich mechanisch zu repetieren. Ein niedrigschwelliger Einstieg und die stufenweise Steigerung des Niveaus machen selbst komplexe Konzepte greifbarer und erhöhen die Selbstwirksamkeitserfahrung von Studierenden.

- Projektbasiertes Lernen: Sukzessives Lernen kann in projektförmigen didaktischen Settings realisiert werden. Lehr-Lern-Projekte mit einem breiten Erkenntnisinteresse, authentischen Problemstellungen und hohem Anwendungsbezug machen die Verbindung zwischen Theorie und Praxis für Studierende erfahrbar. Zudem können in Projekten Kompetenzen anwendungsorientiert geprüft werden.

- Lernen mit echten Daten: Projekte beziehen idealerweise echte Daten ein, die für Studierende von Interesse und Relevanz sind - anstatt sich mit Daten um ihrer selbst willen zu beschäftigen, kann so echte Begeisterung geweckt werden. Zudem sollten Projekte ergebnisoffen angelegt sein. Die intensivierte Auseinandersetzung mit Daten kann Innovationen befördern, Lernerfolge verbessern und die Chancen auf lebenslanges Lernen erhöhen.

\section{Chancen interdisziplinärer Ansätze}

Vieles spricht dafür, dass sich die vorgenannten Gelingensbedingungen besonders gewinnbringend in interdisziplinären Lehr-Lern-Settings realisieren lassen. Schon Ridsdale et al. (2015) haben darauf aufmerksam gemacht, dass die fachübergreifende Zusammenarbeit von Lehrenden für eine systematische Förderung von Data Literacy essenziell ist. Darüber hinaus werden in transdisziplinärer Zusammenarbeit auf organisatorischer und didaktischer Ebene - insbesondere mit hochschulexternen Partner*innen - Mehrwerte für die institutionelle Verankerung der Data Literacy Education an Hochschulen sowie für die Berufsvorbereitung für Studierende aller Fächer gesehen (Ridsdale et al. 2015, S. 18). Auch Schüller et al. (2019) weisen darauf hin, dass Datenprojekte ,ein interdisziplinäres und multiprofessionelles Arbeiten, das die Fähigkeit zum Projektmanagement und die Kenntnis organisatorischer, rechtlicher und ethischer Rahmenbedingungen miteinschließt" (Schüller et al. 2019, S. 25), erfordern. Besonders eindringlich plädieren Kuhn und Krupka (2018) mit Brinda et al. (2019) für die interdisziplinäre Bildung in der digitalen Wissensgesellschaft: 


\begin{abstract}
„Um eine nachhaltige und strukturell verankerte Bildung für die digitale Welt zu gewährleisten, müssen in der akademischen Ausbildung die Erscheinungsformen der Digitalisierung unter verschiedenen Perspektiven betrachtet werden. Jede Erscheinungsform hat sowohl technologische und gesellschaftlich-kulturelle als auch anwendungsbezogene Aspekte, die sich gegenseitig beeinflussen. Daher kann nur deren gemeinsame didaktische Bearbeitung zu einer fundierten und nachhaltigen Bildung führen“ (Kuhn und Krupka 2018, S. 34).
\end{abstract}

Nicht zuletzt wird die Forderung, Interdisziplinarität in der Gestaltung von LehrLern-Angeboten im Kontext der Digitalisierung stärker mitzudenken, auch von Studierenden aller Fächer gestellt (Baumann et al. 2019).

In der Hochschuldidaktik gilt interdisziplinäre Zusammenarbeit längst als essenzieller Faktor für Innovation und Erfolg in Wissenschaft und Wirtschaft (Langewiesche 2005, S. 13). Interdisziplinarität und interdisziplinäre Kompetenzen werden in der vernetzten Welt als Schlüssel für die erfolgreiche Bewältigung der neuen und andersartigen beruflichen Anforderungen angesehen (Kagermann et al. 2013, S. 96). Mit beiden Begriffen ist eine bestimmte Erwartungshaltung assoziiert: Sie gelten sowohl als gesellschaftliche Erfolgsfaktoren als auch als solche Einzelner, insbesondere wenn Kompetenzen mit Interdisziplinarität verbunden sind (Jungert 2013, S. 1; Lerch 2017, S. 19 f.).

Ganz allgemein können interdisziplinäre Kompetenzen als Querschnitt kontextdeterminierter überfachlicher Kompetenzen gefasst werden. Diese ermöglichen durch Transfer erfolgreicher Handlungserfahrungen im Sinne von Schlüsselkompetenzen Handlungsfähigkeit in neuen und komplexen Situationen (Arbeitskreis DQR 2011, S. 8 f.; Lerch 2017, S. 69).

Kompetenzentwicklung vollzieht sich im Handeln (Handlungslernen) durch reflektiertes Erproben und Erfahren, eingebettet in eine soziale Situation (situiertes Lernen) (Dirsch-Weigand und Hampe 2018, S. 37 f.). Kompetenzentwicklung ist zudem Voraussetzung und Ergebnis selbstorganisierten Lernens (Arnold 2010a, S. 173). Selbstorganisiertes Lernen setzt neben der Bereitschaft der Lernenden, sich selbst zu aktivieren und zu motivieren, die Fähigkeit zur Selbstorganisation voraus (Arnold 2010b, S. 264; Quilling 2015, S. 2). Selbstorganisationsfähigkeit meint, dass die Lernenden über jene Reflexions- und Handlungsfähigkeiten verfügen, die es ihnen ermöglichen, ihren eigenen Lernprozess zu gestalten und zu verantworten. $\mathrm{Zu}$ diesen „Erschließungswerkzeugen“ (Arnold 2018, S. 26) gehören insbesondere Methoden und Techniken, den Umgang mit sich selbst, anderen und Lerngegenständen zu reflektieren und zu moderieren (Gerstenmaier und Mandl 2018, S. 225). Zusammenfassend sind Lehr-Lern-Arrangements, die 
Erwerb und Ausbau interdisziplinärer Kompetenzen ermöglichen, auf Selbstorganisation über aktivierende Methoden sowie Projektarbeit an komplexen Problemstellungen ausgerichtet (Lerch 2017, S. 118 ff.; Ricken 2011, S. 64).

Vor diesem Hintergrund eröffnen interdisziplinäre Lehr-Lern-Formate besondere Chancen, Data Literacy zu trainieren. Wenn sich Studierende aus Technik, Informatik, Sozialwesen, Wirtschaft und Design gemeinsam mit komplexen Problemstellungen auseinandersetzen, bringen sie ihre unterschiedlichen fachlichen Perspektiven ein und lernen mit- und voneinander. Dabei erfahren sie anwendungsorientiert, dass für einen planvollen, kritischen und verantwortlichen Umgang mit Daten technische, rechtliche, ökonomische, soziale und ethische Aspekte zusammengedacht werden müssen.

\section{$5 \quad$ Fallstudie: Das Mannheimer Modell Data Literacy Education („,modal“)}

Eine Fallstudie demonstriert mögliche Umsetzungen dieser theoretischen Vorüberlegungen im hochschulischen Kontext. Das Mannheimer Modell Data Literacy Education (,modal“) ermöglicht seit 2018 Studierenden aller Fächer an der Hochschule Mannheim, gemeinsam den planvollen, verantwortlichen und kritischen Umgang mit Daten zu trainieren. Hierfür wurden Lehr-Lern-Angebote für insgesamt 5200 Studierende entwickelt, die an neun Fakultäten 23 unterschiedliche Bachelorstudiengänge studieren. Die Herausforderung und Chance besteht darin, technisch-ingenieurwissenschaftliche, soziale und gestalterische Studiengänge gleichermaßen miteinzubeziehen.

Orientiert an den Data Literacy Frameworks von Ridsdale et al. (2015) und Schüller et al. (2019) wurden spezifische Lernziele von „modal“ operationalisiert, die fünf Kompetenzfeldern zugeordnet werden können: Daten in Beruf und Gesellschaft, Daten sammeln, Daten formen, Daten lesen und Daten leiten Handeln. Diese Systematik übernimmt von Schüller et al. (2019) das Modell der zirkulären Datenwertschöpfung. Außerdem spiegelt sich der Dreiklang der Kompetenzdimensionen Wissen, Fähigkeiten und Haltung in den Lernzielen von „modal“" wider. Die Unterscheidung von drei Niveaustufen der Data Literacy nach Ridsdale et al. (2015) begründet den konsekutiven Aufbau von „modal“.

Drei Stufen ermöglichen Studierenden aller Fächer einen niedrigschwelligen Einstieg und die konsekutive Vertiefung von Data-Literacy-Kompetenzen. Die erste Stufe unimodal ${ }_{l}$ richtet sich an Studienanfänger*innen aller Fächer, die Kernkompetenzen im planvollen, verantwortlichen und kritischen Umgang 
mit Daten entwickeln möchten. Das Format ist als Ringvorlesung mit alternierenden Praxisübungen angelegt. Die zweite Stufe bildet das interdisziplinäre Datenprojekt bimodal 2 . Teilnehmen können Studierende aller Bachelorstudiengänge im Grundstudium. In fachlich gemischten Kleingruppen werden fortgeschrittene Data-Literacy-Kompetenzen praxisnah trainiert. Diese werden im Sinne der Entwicklung von Future Skills mit sogenannten klassischen, „nicht digitalen Schlüsselkompetenzen“ wie Teamfähigkeit, Adaptionsfähigkeit, Kreativität und Durchhaltevermögen anwendungsorientiert verzahnt (Kirchherr et al. 2018, S. 4 ff.). Die dritte Stufe trimodal 3 richtet sich an Studierende im Hauptstudium. Die Teilnehmer*innen entwickeln Data-Literacy-Kompetenzen auf Expert*innenniveau. In Kooperation mit Praxispartner*innen werden transdisziplinäre datengetriebene Lehr-Lern-Forschungsprojekte realisiert (Nagel 2020). Über die Begleitsäule multimodal ${ }_{n}$ werden unter anderem Kooperationen mit hochschulexternen Praxispartner*innen und der Transfer von Projektergebnissen sowie Evaluation und Wirksamkeitsforschung organisiert.

Wie ist „modal“ in Studium und Lehre an der Hochschule Mannheim curricular verankert? Das interdisziplinäre Datenprojekt bimodal 2 wird für Studierende im Grundstudium in allen 23 Bachelorstudiengängen im Rahmen von Wahlpflichtbereichen als Studienleistung anerkannt und mit zwei bis vier ECTS-Punkten kreditiert. Die Lehr-Lern-Angebote auf den beiden anderen Stufen sind in einigen Studiengängen anrechenbar. Zusätzlich wird die Teilnahme an allen Formaten zertifiziert.

Die didaktische Feinjustierung lässt sich besonders instruktiv am interdisziplinären Datenprojekt auf der zweiten Stufe bimodal 2 veranschaulichen. Die Ausgestaltung des Lehr-Lern-Settings orientiert sich an den von Ridsdale et al. (2015) und Schüller et al. (2019) zusammengetragenen Gelingensbedingungen. Außerdem wurden weitere hochschuldidaktische Best Practices wie das i-LEARN Modell herangezogen, die sich in der Entwicklung von Datenkompetenzen als erfolgreich erwiesen haben (Greenwell 2019; Neuman 2016, S. 274 ff.). Im Fokus stehen die Ermöglichung selbstorganisierten Lernens, ein hoher Anwendungsbezug und die Erfahrung von Selbstwirksamkeit. Die folgenden sieben Bausteine tragen dazu bei, dieses Ziel bei bimodal 2 zu erreichen.

1. Lernen in heterogenen Kleingruppen: Die Teilnehmer*innen am Datenprojekt werden in interdisziplinären Kleingruppen zusammengeführt. Diese fachliche und soziokulturelle Vielfalt in ihrem Team wird als Chance erlebt. Die Heterogenität der Studierenden birgt große Potenziale für ein gemeinsames Lernen mit- und voneinander. Insbesondere entdecken die Studierenden unterschiedliche Ansätze und Methoden im Umgang mit Daten. 
2. Projektförmiges Lernen: Die bimodal 2 -Teams entwickeln semesterbegleitend eigene Projekte innerhalb einer offenen Themenstellung. Die projektförmige Zusammenarbeit fordert und fördert Selbstorganisation. Studierende identifizieren Problemfelder selbst, legen gemeinsam Ziele und Arbeitsschritte fest und entscheiden eigenverantwortlich über Methoden zur Entwicklung eines Lösungskonzepts. Zum Abschluss treten die Teams in einem Wettbewerb gegeneinander an und pitchen ihre Projekte im Rahmen einer öffentlichen Veranstaltung.

3. Offene Themenstellung, echte Daten: Die praxisnahe Themenstellung wird weit und ergebnisoffen gefasst, um Anknüpfungspunkte für möglichst viele Disziplinen zu bieten. Beispielsweise stand der bimodal 2 -Durchlauf im Sommer 2019 unter dem Motto „Zusammen Leben in Mannheim“. Gut 40 Teilnehmer*innen beschäftigten sich mit Mietpreisentwicklung, Gestaltung des öffentlichen Raums und gesellschaftlicher Integration in ihrer Studienstadt. Im Winter 2019/20 lautete das Thema „klima:freundlich - Hochschule Mannheim“. Über 85 Studierende interessierten sich für die Klimabilanz ihrer Mensa, regenerative Energien für Hörsaalgebäude oder Klimafolgenanpassung auf dem Campus. Über einen Bezug zur Lebenswelt der Studierenden laden die Themen zur Auseinandersetzung mit großen gesellschaftlichen Herausforderungen ein. Den Teams werden kuratierte Datensätze zur Verfügung gestellt, mit denen sie den Problemraum erkunden und erste Ideen finden können. Kommunale Open-Data-Portale wie zum Beispiel der Städte Hamburg (Staatsarchiv Hamburg o. J.) oder Mannheim (Gemeinderat der Stadt Mannheim 2015) bieten eine reichhaltige Quelle unter anderem für Bevölkerungsstatistiken, Geodaten oder Klimainformationen. Eigene Datenerhebungen der Studierenden unterstützen die Entwicklung ganzheitlicher Lösungsansätze. Die Teams werden angeregt, ihre Entwürfe mithilfe digitaler Technologien, zum Beispiel Virtual Reality, umzusetzen.

4. Peer-Learning: Jedes bimodal 2 -Team wird von studentischen Peer-to-PeerTutor*innen begleitet. Für diese Aufgabe werden Studierende im Hauptstudium aus allen Fächern gewonnen. Beim interdisziplinären Datenprojekt moderieren sie gruppendynamische Prozesse und geben Hilfe zur Selbsthilfe. Auf diese Aufgabe werden die Peer-Tutor*innen in einem fünftägigen Training vorbereitet. Peer-Learning hat sich als motivierendes und die Selbstorganisation stärkendes Setting bewährt (Bandtel und Trefs 2019).

5. Flipped Classroom: Inter- und transdisziplinäre Kooperation vertiefen den Anwendungsbezug. Professor*innen aller Fakultäten und Mitarbeiter*innen zentraler Einrichtungen unterstützen die bimodal 2 -Teams in fachlichen Fragen. Diese Form der Interaktion dreht den Klassenraum um: Lernende arbeiten 
sich eigenständig in ein Thema ein und begegnen Lehrenden auf Augenhöhe. Zudem stehen Kooperationspartner*innen aus Wirtschaft, Verwaltung und Zivilgesellschaft den Studierenden mit Expertise und tatkräftiger Unterstützung zur Seite.

6. Workshops und Reallabore: Werkzeuge für den planvollen, verantwortlichen und kritischen Umgang mit Daten erarbeiten bimodal 2 -Teilnehmer*innen in Workshops. Im Rahmen der Skill Week durchlaufen Mitglieder aller Teams Einführungen in Datenrecherche, Datenerhebung, Statistik und Datenanalyse mit R, Datenvisualisierung sowie Ergebnispräsentation. Die Workshops werden von Lehrenden aus den Fakultäten für Informatik, Sozialwesen, Kommunikationsdesign und Maschinenbau sowie von der Bibliothek der Hochschule Mannheim durchgeführt. Das Jigsaw- oder Gruppenpuzzleformat sorgt dafür, dass Studierende die Datenwerkzeuge nicht nur kennenlernen, sondern auch aktiv an die anderen Teammitglieder weitergeben. Der Wechsel in die Rolle von Expert*innen unterstützt die vertiefte Verarbeitung der Lerninhalte (eteaching.org 2015). Ziel der Skill Week ist, den Studierenden Methoden der Datensammlung, -auswertung und -visualisierung an die Hand zu geben, die sie im Sinne eines (Real-)Labors bei der inter- und transdisziplinären Projektarbeit im Team anwendungsorientiert einsetzen können.

7. Virtual Reality in der Lehre: Mit der Cave Automatic Virtual Environment (CAVE) steht bimodal 2 -Teilnehmer*innen eine 3D-Entwicklungsumgebung für Virtual Engineering zur Verfügung: Wände, Boden und Decke des würfelförmigen Raumes werden von zehn Hochleistungsprojektoren mit stereoskopischen Daten bespielt. So können virtuelle Welten begeh- und erlebbar gemacht werden. bimodal ${ }_{2}$-Teams nutzen die CAVE, um Daten zu explorieren sowie um virtuelle Prototypen zu konstruieren und zu testen. Dabei lernen die Studierenden zum einen niedrigschwellig hochaktuelle Entwicklungsprozesse und Technologien kennen. Zum anderen stimuliert die visuelle Umsetzung den planvollen, verantwortlichen und kritischen Umgang mit Daten, da Zusammenhänge an konkreten Objekten sichtbar gemacht und diskutiert werden können. Vor allem aber wirkt sich die Erfahrung, selbst komplexe Konzepte virtuell realisieren zu können, positiv auf Selbstwirksamkeit und Teamerleben aus.

\section{$6 \quad$ Evaluation und Kompetenzmessung}

Gerade in der Erprobungsphase innovativer Lehr-Lern-Formate ist die engmaschige Evaluation von hoher Bedeutung. Im Mannheimer Modell Data Literacy Education kommen formative und summative Verfahren sowie standardisierte 
und qualitative Instrumente zum Einsatz. Als besonders gewinnbringend haben sich regelmäßige Feedbackrunden mit Tutor*innen erwiesen. In teilstrukturierten Fokusgruppen mit offener Gesprächsatmosphäre können sehr feingliedrig Erkenntnisse über Studierendenzufriedenheit, Lernerfolge sowie Weiterentwicklungsbedarf der Lehr-Lern-Angebote gewonnen werden.

Darüber hinaus wird die Wirksamkeit der Maßnahmen über Self-Assessments der Teilnehmer*innen gemessen. Dabei werden zum einen Selbsteinschätzungen bestimmter Persönlichkeitsmerkmale erhoben, die im Kontext der Entwicklung von Future Skills von Interesse sind, weil sie Rückschlüsse auf Motivation, Selbstorganisationsfähigkeit und Teamwork zulassen. Beispielsweise bringt das Ausmaß an internaler und externaler Kontrollüberzeugung zum Ausdruck, inwieweit Studierende Ereignisse als Konsequenzen ihres eigenen Handelns begreifen oder als Resultat äußerer Umstände (Kovaleva et al. 2014). Mit der allgemeinen Selbstwirksamkeit lässt sich die Einschätzung der eigenen Kompetenzen beschreiben, Schwierigkeiten im Alltag erfolgreich zu meistern (Beierlein et al. 2014a). Anhand des selbst zugeschriebenen interpersonalen Vertrauens wird deutlich, wie sehr Lernende bereit sind, sich auf andere zu verlassen. Das erlaubt Aussagen über die Fähigkeit, sich auf neuartige und kontingente Herausforderungen einzustellen (Beierlein et al. 2014b). Zum anderen werden mit dem Kompetenzraster von Eichhorn und Tillmann (2018) digitale Kompetenzen aller Teilnehmer*innen auf sechs Subdimensionen erfasst. Alle eingesetzten Skalen erfüllen teststatistische Gütekriterien. Die Erhebung erfolgt jeweils zu Beginn und zum Ende des Semesters über einen Onlinefragebogen. Im Prä-Post-Design werden Mittelwertvergleiche angestellt.

Exemplarisch werden hier einige Befunde der Erhebung aus dem interdisziplinären Datenprojekt bimodal 2 vom Wintersemester 2019/20 diskutiert. An der Eingangsbefragung haben 43, an der Ausgangsbefragung 45 Studierende teilgenommen. Beim Blick auf die gemessenen Persönlichkeitsmerkmale (Abb. 1) fallen unmittelbar die insgesamt hohen Ausprägungen an Selbstwirksamkeit ( $m_{\text {Eingang }}=4,02 ; m_{\text {Ausgang }}=3,93$; fünfpolige Antwortskala) und internaler Kontrollüberzeugung ( $\left.m_{\text {Eingang }}=4,21 ; m_{\text {Ausgang }}=4,20\right)$ sowie korrespondierend die geringen Werte für externale Kontrollüberzeugung $\left(m_{\text {Eingang }}=2,01 ; m_{\text {Ausgang }}=\right.$ $2,29)$ auf, die sich die Teilnehmer*innen selbst zuschreiben. Das Maß an interpersonalem Vertrauen liegt fast exakt auf dem Skalenmittelwert $\left(m_{\text {Eingang }}=3,03\right.$; $m_{\text {Ausgang }}=3,04$ ).

Der Vergleich zwischen beiden Erhebungswellen zeigt nahezu unveränderte Mittelwerte bei der Eingangs- und Ausgangsbefragung. Wie erwartet erweisen sich also Persönlichkeitsmerkmale als sehr stabil. Die Selbsteinschätzungen lassen darauf schließen, dass das freiwillige Lehr-Lern-Angebot insbesondere jene 


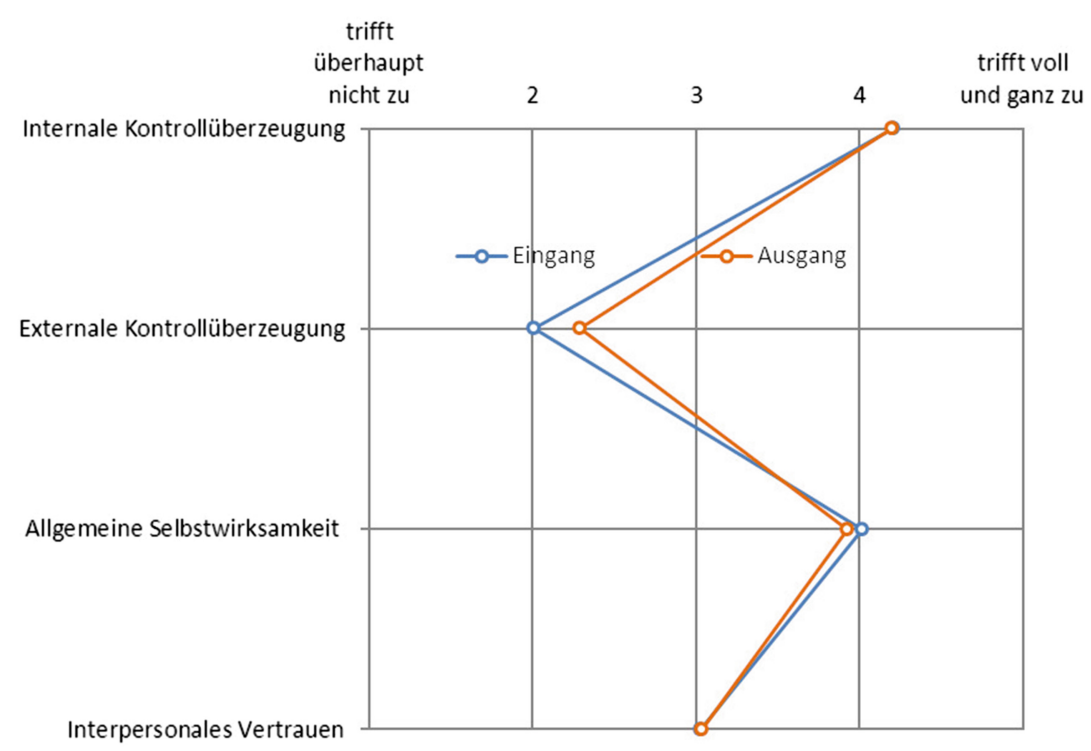

Abb. 1 Kontrollüberzeugungen, Selbstwirksamkeit und interpersonales Vertrauen der Teilnehmer*innen am interdisziplinären Datenprojekt im Winter 2019/20 ( $\mathrm{n}_{\text {Eingang }}=43 ; \mathrm{n}_{\text {Ausgang }}$ = 45; Instrumente: IE-4 (Kovaleva et al. 2014); ASKU (Beierlein et al. 2014a); KUSIV (Beierlein et al. 2014b); fünfpolige Antwortskalen). (Eigene Darstellung)

Studierende attrahiert, die bereits im Vorfeld über hohe Kompetenzerwartungen verfügen, mit Schwierigkeiten und Hindernissen umgehen zu können. Für die Weiterentwicklung der Lehr-Lern-Angebote leitet sich daraus die Herausforderung ab, das Format dergestalt anzupassen, dass auch Studierende angesprochen werden, die von der Förderung „klassischer Schlüsselkompetenzen“ im Kontext der Future Skills besonders profitieren würden.

Ein ganz anderes Bild zeigt sich bei den Selbsteinschätzungen von Datenkompetenzen (Abb. 2). In der Eingangsbefragung zum Wintersemester 2019/20 gaben sich die Teilnehmer*innen im Mittel auf drei von sechs gemessenen Dimensionen Werte um den Skalenmittelwert oder deutlich darunter. Insbesondere auf der Subskala „Digitale Wissenschaft“ ( $m_{\text {Eingang }}=3,00$; sechspolige Antwortskala) fielen die Selbsteinschätzungen auffallend niedrig aus. Demzufolge sahen die Studierenden ihre Kompetenzen in der „Nutzung und Erzeugung digitaler Daten, Quellen, Methoden und Publikationen, um wissenschaftliche Ziele zu erreichen“ 


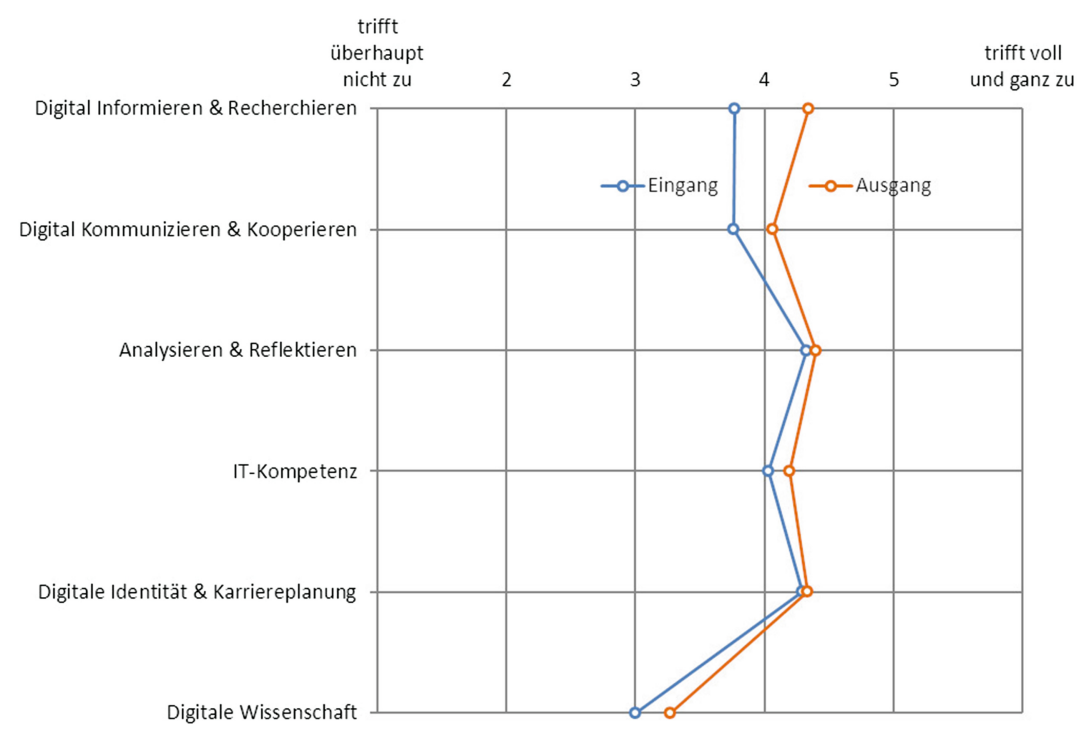

Abb. 2 Datenkompetenzen der Teilnehmer*innen am interdisziplinären Datenprojekt im Winter 2019/20 ( $\mathrm{n}_{\text {Eingang }}=43 ; \mathrm{n}_{\text {Ausgang }}=45$; Instrument: , Kompetenzraster digitale Kompetenz" (Eichhorn und Tillmann 2018); ausgewählte Dimensionen und Items; sechspolige Antwortskala). (Eigene Darstellung)

(Eichhorn und Tillmann 2018, S. 70), als gering ausgeprägt an. Etwas höhere Eingangswerte gaben sich die Studierenden auf den Dimensionen „Digitale Identität und Karriereplanung" ( $m_{\text {Eingang }}=4,29$ ) sowie "Analysieren und Reflektieren“ $\left(m_{\text {Eingang }}=4,33\right)$. Das heißt, sie empfanden sich schon zu Beginn des interdisziplinären Datenprojekts als relativ kompetent hinsichtlich „Aufbau, Pflege und Schutz einer eigenen digitalen Identität“ (Eichhorn und Tillmann 2018, S. 70) und schrieben sich einige Kenntnisse und Fähigkeiten für eine ,[e]ffiziente und kritische Nutzung digitaler Medien, Analyse und Kritik des eigenen Medieneinsatzes“ (Eichhorn und Tillmann 2018, S. 70) zu.

Zum Ende des Semesters konnten auf allen sechs gemessenen Dimensionen des Kompetenzrasters höhere Mittelwerte als zu Beginn beobachtet werden. Die größte Differenz zwischen Eingangs- und Ausgangsbefragung zeigte sich auf der Subskala „Digital informieren und recherchieren“ $\left(m_{\text {Eingang }}=3,77\right.$; $\left.m_{\text {Ausgang }}=4,34\right)$. Das heißt, zum Abschluss des interdisziplinären Datenprojekts 
empfanden die Teilnehmer*innen ihre Kompetenzen im Umgang mit Suchinstrumenten, Suchstrategien, Literaturverwaltung, Wissensmanagement, Urheberrecht und Datenschutz im Mittel als ausgeprägter denn zu Beginn. Auch auf der Dimension „Digital kommunizieren und kooperieren“ ( $m_{\text {Eingang }}=3,76 ; m_{\text {Ausgang }}$ $=4,07)$ zeigte sich eine deutlich positive Veränderung. Offensichtlich haben die Studierenden im Semesterverlauf eine größere Sicherheit mit den Themen Onlinecommunitys, Web 2.0, Social Media, Open Source, Open Access und Lernplattformen erlangt. Nicht zuletzt ließ sich auch auf der Subskala mit dem niedrigsten Eingangswert, „Digitale Wissenschaft“, ein leichter „Aufwärtstrend“ ablesen ( $m_{\text {Eingang }}=3,00 ; m_{\text {Ausgang }}=3,27$ ). Es bleibt jedoch festzuhalten, dass die Ausgangsbefragung auf allen gemessenen Dimensionen noch erhebliche Entwicklungspotenziale der digitalen Kompetenzen offenbarte. Daraus lässt sich zum einen unmittelbar der Bedarf ableiten, entsprechende Lehr-Lern-Angebote nicht nur einmalig und punktuell, sondern mit steigendem Anspruchsniveau wiederholt im Studienverlauf zu platzieren. Zum anderen muss es bei künftigen Weiterentwicklungen der Lehr-Lern-Programme darum gehen, Data Literacy noch tiefer in die Curricula aller Fächer zu integrieren (Abschn. 3).

Selbstverständlich sind die diskutierten Befunde aufgrund der geringen Fallzahlen und zahlreicher nicht kontrollierbarer Einflussfaktoren mit großer Vorsicht zu interpretieren. Sie deuten jedoch darauf hin, dass Studierende einen ausgeprägten Bedarf bei der Entwicklung digitaler Kompetenzen wahrnehmen, der durch bestehende curriculare Lehr-Lern-Angebote nicht gedeckt wird. Die dabei zum Ausdruck kommende Selbstreflexionsfähigkeit ist grundsätzlich positiv zu beurteilen - inhaltlich jedoch ist der Befund alarmierend. In künftigen Untersuchungen gilt es zu beobachten, inwieweit sich diese Tendenz bestätigt. Schon jetzt allerdings zeichnet sich ab, dass das Engagement zur Förderung von Data Literacy für Studierende aller Fächer weiter intensiviert werden muss.

\section{Erkenntnisse für die (Weiter-)Entwicklung von Data-Literacy-Lehr-Lern-Angeboten}

Data Literacy ist eine Schlüsselkompetenz des 21. Jahrhunderts (Schüller et al. 2019, S. 10). Mehr denn je stehen Hochschulen in der Verantwortung, Studierenden aller Fächer den planvollen, verantwortlichen und kritischen Umgang mit Daten zu ermöglichen, um sie bestmöglich auf die mündige Teilhabe und aktive Mitgestaltung der digitalen Gesellschaft vorzubereiten.

Die Ergebnisse der Fallstudie (Abschn. 5) und der Begleitforschung zum Mannheimer Modell Data Literacy Education (Abschn. 6) deuten darauf hin, 
dass niedrigschwellige interdisziplinäre Lehr-Lern-Angebote $\mathrm{zu}$ einer positiven Entwicklung von Datenkompetenzen Studierender aller Fächer beitragen können. Wichtig sind ein konsekutiver Aufbau entsprechender Programme mit steigendem Anspruchsniveau sowie der Einsatz innovativer Didaktiken. Allerdings hat sich gezeigt, dass extracurriculare Angebote vor allem diejenigen Studierenden attrahieren, die bereits für die Relevanz von Datenkompetenzen sensibilisiert sind.

Die Herausforderung liegt daher in der nachhaltigen Integration geeigneter Lehr-Lern-Angebote in die Curricula aller Fächer. Dabei erweist sich die begriffliche Offenheit von Data Literacy als Chance. So kann das Konzept einerseits agil an den sich wandelnden Bedarf von Wissenschaft, Wirtschaft und Gesellschaft angepasst werden (Ridsdale et al. 2015, S. 12). Andererseits eröffnet die integrative Definition vielfältige Anknüpfungspunkte an Lehr-Lern-Veranstaltungen, die an Hochschulen bereits angeboten werden. Auf diese Weise können Maßnahmen zur Entwicklung von Datenkompetenzen übergangsweise relativ niedrigschwellig in bestehende Studiengänge eingebunden werden (Ridsdale et al. 2015, S. 19 ff.). Ansätze, die interdisziplinäre Grundlagen und fachliche Spezialisierung miteinander kombinieren, erweisen sich dabei als besonders geeignet, Studierenden die Entwicklung von Future Skills zu ermöglichen (Heidrich et al. 2018).

Aktuell gibt es an vielen Hochschulen Planungen, das interdisziplinäre LehrLern-Angebot weiter auszubauen und hierfür entsprechende Fenster in der Modulstruktur aller Studiengänge zu schaffen. Diese Entwicklung eröffnet die Chance, Data Literacy Education für Studierende aller Fächer curricular zu integrieren. Um dieses Ziel zu erreichen, ist der fach- und standortübergreifende Erfahrungsaustausch unter Lehrenden und Lernenden von hohem Wert. Denn gerade bei Fragen der institutionellen Verankerung und didaktischen Ausgestaltung von Data Literacy Education liegt der Schlüssel im gemeinsamen mit- und voneinander Lernen.

\section{Literatur}

Arbeitskreis DQR. (2011). Deutscher Qualifikationsrahmen für lebenslanges Lernen. https:// www.dqr.de/. Zugegriffen: 30. Jan. 2019.

Arnold, R. (2010a). Kompetenz. In R. Arnold, S. Nolada, \& E. Nuissl (Hrsg.), Wörterbuch Erwachsenenbildung, (2. Aufl., S. 172-173). Bad Heilbrunn: Klinkhardt.

Arnold, R. (2010b). Selbstorganisation - Selbststeuerung. In R. Arnold, S. Nolada, \& E. Nuissl (Hrsg.), Wörterbuch Erwachsenenbildung, (2. Aufl., S. 263-265). Bad Heilbrunn: Klinkhardt.

Arnold, R. (2018). Wie man lehrt, ohne zu belehren: 29 Regeln für eine kluge Lehre. Das LENA-Modell. Heidelberg: Carl-Auer. 
Bandtel, M., \& Trefs, L. (2019). Ermöglichungsräume für selbstorganisiertes Lernen: Wollen, Können, Gefragt werden. In Y. Berkle, H. Hettrich, K. Kilian, \& J. Woll (Hrsg.), Visionen von Studierenden-Erfolg (S. 229-244). Kaiserslautern: Hochschule Kaiserslautern.

Baumann, J., Böckel, A., Denker, F., Gross, P., Kern, E., Lamprecht, M., Reimann, J., Rensinghoff, B., Sari, Z., Schopf, E., Wächtler, E., \& Meyer, H. (2019). The Digital Turn aus Studierendenperspektive. Studentisches Thesenpapier zur Digitalisierung in der Hochschulbildung. Berlin: Hochschulforum Digitalisierung. https://doi.org/10.5281/zenodo. 3484574.

Beierlein, C., Kemper, C. J., Kovaleva, A., \& Rammstedt, B. (2014). Interpersonales Vertrauen (KUSIV3). Zusammenstellung sozialwissenschaftlicher Items und Skalen (ZIS). https:// doi.org/10.6102/zis37.

Beierlein, C., Kovaleva, A., Kemper, C. J., \& Rammstedt, B. (2014). Allgemeine Selbstwirksamkeit Kurzskala (ASKU). Zusammenstellung sozialwissenschaftlicher Items und Skalen (ZIS). https://doi.org/10.6102/zis35.

Brinda, T., Brüggen, N., Diethelm, I., Knaus, T., Kommer, S., Missomelius, P., Leschke, R., Tilemann, F., \& Weich, A. (2019). Frankfurt-Dreieck zur Bildung in der digital vernetzten Welt. Ein interdisziplinäres Modell. merz - medien + erziehung. zeitschrift für medienpädagogik, 4, 1-9.

Bundesregierung. (2019). Digitalisierung gestalten. Umsetzungsstrategie der Bundesregierung. Berlin: Presse- und Informationsamt der Bundesregierung. https://www.bundesreg ierung.de/breg-de/themen/digital-made-in-de. Zugegriffen: 28. Apr. 2020.

Dirsch-Weigand, A., \& Hampe, M. (2018). Interdisziplinäre Studienprojekte gestalten. Aus der Praxis für die Praxis. Bielefeld: W. Bertelsmann.

e-teaching.org. (2015). Gruppenpuzzle. https://www.e-teaching.org/didaktik/konzeption/met hoden/gruppenlernen/puzzle. Zugegriffen: 3. Juli 2020.

Eichhorn, M., \& Tillmann, A. (2018). Digitale Kompetenzen von Hochschullehrenden messen. Validierungsstudie eines Kompetenzrasters. In D. Krömker \& U. Schroeder (Hrsg.), DeLFI 2018 - Die 16. E-Learning Fachtagung Informatik (S. 69-80). Bonn: Gesellschaft für Informatik e. V.

Engel, J., Biehler, R., Frischemeier, D., Podworny, S., Schiller, A., \& Martignon, L. (2019). Zivilstatistik: Konzept einer neuen Perspektive auf Data Literacy und Statistical Literacy. AStA Wirtschafts- und Sozialstatistisches Archiv, 13, 213-244.

Gemeinderat der Stadt Mannheim. (2015). Open Data Portal der Stadt Mannheim. https:// mannheim.opendatasoft.com/page/home/. Zugegriffen: 15. Okt. 2020.

Gerstenmaier, J., \& Mandl, H. (2018). Konstruktivistische Ansätze in der Erwachsenenbildung und Weiterbildung. In R. Tippelt \& A. von Hippel (Hrsg.), Handbuch Erwachsenenbildung/Weiterbildung (6. Aufl., S. 221-234). Wiesbaden: Springer VS.

Greenwell, S. (2019). The I-LEARN Model: Introduction. https://libguides.uky.edu/c.php? $\mathrm{g}=223353 \& \mathrm{p}=1479162$. Zugegriffen: 21. Apr. 2020.

Heidrich, J., Bauer, P., \& Krupka, D. (2018). Future Skills: Ansätze zur Vermittlung von Data Literacy in der Hochschulbildung (Arbeitspapier, 37). Berlin: Hochschulforum Digitalisierung. https://doi.org/10.5281/zenodo.1413119.

Jungert, M. (2013). Was zwischen wem und warum eigentlich? Grundsätzliche Fragen der Interdisziplinarität. In M. Jungert, E. Romfeld, \& T. Sukopp (Hrsg.), Interdisziplinarität. Theorie, Praxis, Probleme (S. 1-12). Darmstadt: WBG. 
Kagermann, H., Wahlster, W., \& Helbig, J. (2013). Deutschlands Zukunft als Produktionsstandort sichern. Umsetzungsempfehlungen für das Zukunftsprojekt Industrie 4.0. Frankfurt a. M.: Geschäftsstelle der Plattform Industrie 4.0.

Kirchherr, J. W., Klier, J., Lehmann-Brauns, C., \& Winde, M. (2018). Future Skills: Welche Kompetenzen in Deutschland fehlen. Future Skills-Diskussionspapier, 1. Essen: Stifterverband für die Deutsche Wissenschaft e. V.

Kovaleva, A., Beierlein, C., Kemper, C. J., \& Rammstedt, B. (2014). Internale-ExternaleKontrollüberzeugung-4 (IE-4). Zusammenstellung sozialwissenschaftlicher Items und Skalen (ZIS). https://doi.org/10.6102/zis184.

Kuhn, S., \& Krupka, D. (2018). Data Literacy Education. Interdisziplinäre Bildung für die digitale Wissensgesellschaft. Synergie Fachmagazin für Digitalisierung in der Lehre, 6, 32-36.

Langewiesche, D. (2005). Einsamkeit und Gespräch. Hoffnungen eines Geisteswissenschaftlers bei der Gründung des Max-Weber-Kollegs. In H. Joas \& H. G. Kippenberg (Hrsg.), Interdisziplinarität als Lernprozeß. Erfahrungen mit einem handlungstheoretischen Forschungsprogramm (S. 13-21). Göttingen: Wallstein.

Lerch, S. (2017). Interdisziplinäre Kompetenzen. Eine Einführung. Münster: Waxmann.

Lübcke, M., \& Wannemacher, K. (2018). Vermittlung von Datenkompetenzen an den Hochschulen: Studienangebote im Bereich Data Science. Forum Hochschulentwicklung. Hannover: HIS-Institut für Hochschulentwicklung.

Nagel, T. (2020). Visually analysing urban mobility: Results and insights from three student research projects. KN - Journal of Cartography and Geographic Information, 70, 11-18.

Neuman, D. (2016). Toward a theory of information literacy: Information science meets instructional systems design. In S. Kurbanoğlu, J. Boustany, S. Špiranec, E. Grassian, D. Mizrachi, L. Roy, \& T. Çakmak (Hrsg.), Information literacy: Key to an inclusive society (S. 267-276). Cham: Springer International Publishing.

Pothier, W., \& Condon, P. B. (2019). Towards data literacy competencies: business students, workforce needs, and the role of the Librarian. Journal of Business \& Finance Librarianship, 25:3-4, 1-24.

Quilling, K. (2015). Ermöglichungsdidaktik. Der DIE-Wissensbaustein für die Praxis. DIE space Wissensbaustein. www.die-bonn.de/wb/2015-ermoeglichungsdidaktik-01.pdf. Zugegriffen: 27. Nov. 2018.

Ricken, J. (2011). Universitäre Lernkultur. Fallstudien aus Deutschland und Schweden. Wiesbaden: VS Research.

Ridsdale, C., Rothwell, J., Smit, M., Ali-Hassan, H., Bliemel, M., Irvine, D., Kelley, D., Matwin, S., \& Wuetherick, B. (2015). Strategies and best practices for data literacy education, knowledge synthesis report. Halifax: Dalhousie University. https://dataliter acy.ca/. Zugegriffen: 2. Mai 2018.

Schüller, K., Busch, P., \& Hindinger, C. (2019). Future Skills: Ein Framework für Data Literacy. Kompetenzrahmen und Forschungsbericht (Arbeitspapier, 47). Berlin: Hochschulforum Digitalisierung. https://doi.org/10.5281/zenodo.3349865.

Staatsarchiv Hamburg (o. J.). Open data Hamburg. Transparenzportal Hamburg. https://tra nsparenz.hamburg.de/open-data/. Zugegriffen: 16. Okt. 2020. 
Open Access Dieses Kapitel wird unter der Creative Commons Namensnennung 4.0 International Lizenz (http://creativecommons.org/licenses/by/4.0/deed.de) veröffentlicht, welche die Nutzung, Vervielfältigung, Bearbeitung, Verbreitung und Wiedergabe in jeglichem Medium und Format erlaubt, sofern Sie den/die ursprünglichen Autor(en) und die Quelle ordnungsgemäß nennen, einen Link zur Creative Commons Lizenz beifügen und angeben, ob Änderungen vorgenommen wurden.

Die in diesem Kapitel enthaltenen Bilder und sonstiges Drittmaterial unterliegen ebenfalls der genannten Creative Commons Lizenz, sofern sich aus der Abbildungslegende nichts anderes ergibt. Sofern das betreffende Material nicht unter der genannten Creative Commons Lizenz steht und die betreffende Handlung nicht nach gesetzlichen Vorschriften erlaubt ist, ist für die oben aufgeführten Weiterverwendungen des Materials die Einwilligung des jeweiligen Rechteinhabers einzuholen.

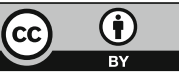

